\section{Tuberculosis associated thrombocytopenic purpura: effectiveness of antituberculous therapy}

\author{
Raphael Borie,, ${ }^{1,2}$ Claire Fieschi, ${ }^{2}$ \\ Eric Oksenhendler, ${ }^{2}$ Lionel Galicier ${ }^{2}$ \\ 'Department of Pneumology A, Hospital \\ Bichat, Assistance Publique, \\ Hôpitaux de Paris, Paris, France \\ 'Department of Clinical Immunology, \\ Hospital St Louis, Assistance Publique, \\ Hôpitaux de Paris, Paris, France
}

\section{Abstract}

Association of immune thrombocytopenic purpura and tuberculosis is a rare condition. In 5 patients presenting with this association, anti-tuberculous therapy was effective on both tuberculosis and thrombocytopenia suggesting a causal relationship between tuberculosis and immune thrombocytopenic purpura

\section{Introduction}

Immune thrombocytopenic purpura (ITP) is characterized by a low platelet count associated with the presence of platelet autoantibodies. The diagnosis of ITP remains a diagnosis of exclusion, and a bone marrow examination should be performed in patients with atypical features. In $5-10 \%$ of cases, ITP is associated with chronic infection (HIV, HCV), systemic autoimmune disorders, lymphoproliferative disorders, and primary immunodeficiency. In adults, ITP is usually chronic (i.e. more than six months in duration). ${ }^{1}$ The response to treatment is defined as an increase in platelet count above $50 \times 10^{9} / \mathrm{L}$, with at least a 2 -fold increase in the initial value and remission is considered if platelet count reaches $150 \times 10^{9} / \mathrm{L}$. ${ }^{2}$

We describe 5 cases of the association of tuberculosis and ITP and the effectiveness of anti-tuberculous therapy (ATT) on both tuberculosis and thrombocytopenia (Figure 1).

\section{Case Reports}

Patients were 3 men and 2 women (Table 1). Median age was 38 years. Major symptoms were pulmonary symptoms in 3 cases and bleeding in 2 cases. However, thrombocytopenia was symptomatic in 4 patients. In 4 of the 5 cases, clinical manifestations of tuberculosis and ITP were concomitant. In one case (patient 3), chest radiography abnormalities were detected four months after the diagnosis of thrombocytopenia. For each patient, ITP diagnosis was based on a platelet count below $30 \times 10^{9} / \mathrm{L}$ and normal bone marrow examination. No patient had splenomegaly. Other secondary forms of ITP were excluded. All patients responded to high-dose intravenous immunoglobulin (HD-IVIg) as in most cases of ITP; ${ }^{2}$ however, none had complete and sustained response. In 3 patients, platelet counts remained below $30 \times 10^{9} / \mathrm{L}$ despite corticosteroid and HD-IVIg. These patients had partial response to danazol or vincristine. Mycobacterium tuberculosis was identified in culture in 3 patients. Histological examination of an adenopathy confirmed the diagnosis in the 2 others. Each strain of mycobacterium tuberculosis was sensitive to conventional therapy. All patients completed treatment and were considered to be cured for tuberculosis. Within two months of anti-tuberculous therapy, all 5 patients were in ITP complete response and were off specific therapy for thrombocytopenia. Patient 2 required corticosteroid for three months for associated hemolytic anemia.

\section{Discussion}

ITP may require treatment with oral prednisone or HD-IVIg. Response rates are almost $75 \%$; however, only $20 \%$ of adult patients with ITP achieve persistent remission. ${ }^{1}$ In the present series, all patients had persistent or refractory ITP. No patient was cured at the time of tuberculosis diagnosis or within the month following the onset of ATT. However, within three months of ATT, ITP was in complete response in all patients. None required further ITP treatment or splenectomy.

Persistence of thrombocytopenia during the first month of ATT may be explained by the fact that Mycobacterium tuberculosis can remain alive in the first two months of anti-tuberculous therapy. ${ }^{3}$ These results suggest a direct relationship between tuberculosis and ITP.

ITP is a rare manifestation of tuberculosis. To our knowledge only 18 reports describing 27 cases of ITP associated with tuberculosis have been reported.$^{46}$ All patients were treated with ATT in combination with corticosteroid, HDIVIg or vincristine. In all cases platelet count recovered with ATT. The correction of thrombocytopenia in all patients within two months of ATT suggests a causal relationship between tuberculosis and thrombocytopenia, although the mechanism remains unknown.

Few causatives conditions may bind thrombocytopenia to tuberculosis. The presence of an underlying disease causative of thrombocytopenia may favor tuberculosis. HIV infection
Correspondence: Raphael Borie, Service de Pneumologie A, Hopital Bichat, 46 rue Henri Huchard,

75877 Paris CEDEX 18, France

E-mail: raphael.borie@bch.aphp.fr

Key words: immune thrombocytopenic purpura immune, peripheral, thrombocytopenia

Acknowledgments: potential conflicts of interest: none. Financial support: none. For this retrospective, observational, non-interventional analysis of medical records, French law does not require the specific approval of an internal review board or the consent of patients.

Received for publication: 14 January 2009

Revision received: 25 February 2009

Accepted for publication: 9 March 2009

This work is licensed under a Creative Commons Attribution 3.0 License (by-nc 3.0).

CCopyright Raphael Borie et al., 2009

Licensee PAGEPress, Italy

Hematology Reviews 2009; 1:e3

doi:10.4081/hr.2009.e3

is a well known cause of ITP and tuberculosis. ${ }^{1}$ Autoimmune disorders and lymphoproliferative diseases, that may cause ITP, are by themselves or when treated, immunosupressive conditions. ${ }^{1}$ None of our patients had such an etiology.

Disseminated tuberculous infection may cause peripheric or central thrombocytopenia. Tuberculous splenic abcess is rare but a possible cause of hypersplenism and peripheric thrombocytopenia. None of our patients had splenomegaly. All patients had bone marrow aspiration to confirm peripheric thrombocytopenia, while bone marrow involvement of tuberculosis may provoke central thrombocytopenia. Hemophagocytic lymphohistiocytosis is an uncontrolled activation of $\mathrm{T}$ cells and macrophages, and an overproduction of inflammatory cytokines responsible for thrombocytopenia and anemia. It has been previously decribed secondary to tuberculosis. ${ }^{7}$ However, bone marrow aspirate excluded this diagnosis.

Appearance of thrombocytopenia after treatment of tuberculosis is classically induced by rifampicin and recovered after stopping rifampicin. ${ }^{8}$ On the contrary, development of tuberculosis after long-term steroid treatment is not uncommon while steroids are immunosuppresive. One of our patients (case 3) had received corticoid before the diagnosis of tuberculosis was made. At first she was asymptomatic and chest radiography was considered as normal. But four months later, she was coughing and dyspneic; chest radiography revealed abnormalities and Ziehl coloration of the sputum was positive for BAAR. The short 
Table 1. Characteristics of the 5 patients with immune thrombocytopenic purpura and tuberculosis.

\begin{tabular}{|c|c|c|c|c|c|c|}
\hline Patient & Age/Sex & $\begin{array}{l}\text { Delay between } \\
\text { ITP and } \\
\text { tuberculosis }\end{array}$ & Symptoms & $\begin{array}{l}\text { Tuberculosis } \\
\text { CT scan }\end{array}$ & Diagnosis & $\begin{array}{l}\text { Duration of } \\
\text { treatment }\end{array}$ \\
\hline 1 & $56 / \mathrm{M}$ & None & Pulmonary & $\begin{array}{l}\text { Tree in bud pattern } \\
\text { bronchiolitis of the } \\
\text { upper lobes }\end{array}$ & Sputum culture & 6 months \\
\hline 2 & $16 / \mathrm{F}$ & None & Asthenia & $\begin{array}{l}\text { Retroperitoneal } \\
\text { adenopathy }\end{array}$ & $\begin{array}{l}\text { Biopsy of the } \\
\text { adenopathy }\end{array}$ & 9 months \\
\hline 3 & $40 / F$ & 4 months & Pulmonary & $\begin{array}{l}\text { Cavernous } \\
\text { destruction of the } \\
\text { left upper lung }\end{array}$ & Sputum culture & 6 months \\
\hline 4 & $38 / \mathrm{M}$ & None & Fever & $\begin{array}{l}\text { Pulmonary } \\
\text { micronodules and } \\
\text { mediastinal adenopathies }\end{array}$ & $\begin{array}{l}\text { Biopsy of an } \\
\text { adenopathy }\end{array}$ & 6 months \\
\hline 5 & $26 / M$ & None & Pulmonary & $\begin{array}{l}\text { Cavernous destruction } \\
\text { of both upper lobes }\end{array}$ & Sputum culture & 6 months \\
\hline
\end{tabular}

F, female; $M$, male.

Patient 1

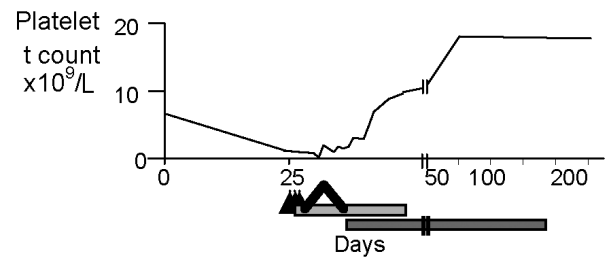

Patient 3

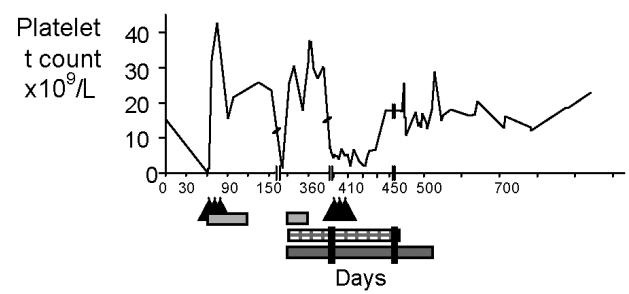

Patient 5

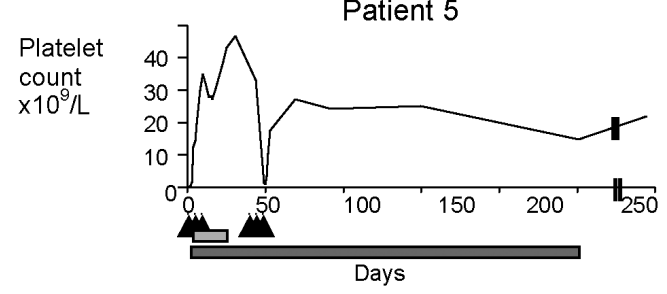

Patient 2

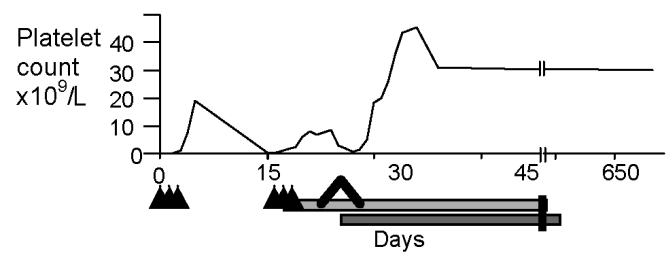

Patient 4

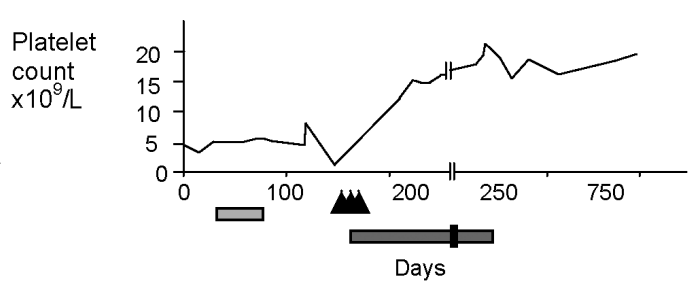

Figure 1. Platelet counts in 5 patients presenting idiopathic thrombocytopenic purpura concommitant to tuberculosis. Thrombocytopenia resolved within three months of antituberculous therapy. course of steroids (less than a month) and the brief period between steroid treatment and the discovery of radiographic abnormalities suggest that active tuberculosis was already present when steroids were prescribed and that the treatment only favored progression of tuberculosis. In all other cases, active tuberculosis and thrombocytopenia were concomitant.

The pathophysiology of thrombopenia in tuberculosis remains unanswered. This is a rare condition, estimated to occur in less than $1 \%$ of cases of tuberculosis. Mycobacterium tuberculosis may share antigene with platelet leading to antiplatelet antibody formation. Specific HLA presentation of tuberculosis could also lead to antiplatelet immunity response in some patients. ${ }^{1}$ However, only one patient developed an auto-antibody. In this case, these were anti-hematia antibodies revealed with Coombs testing. None of our patients test- ed positive for antinuclear antibodies.

Tuberculosis is a rare but curative cause of thrombocytopenia. Thrombocytopenic patients, and particularly chronic and hard to treat ITP, should benefit from tuberculosis depistage.

In cases of tuberculosis associated ITP, recurrence of thrombocytopenia is frequent in the first two months, and patients may benefit from close observation leading to a continuation of ITP specific therapy. 


\section{References}

1. Cines DB, Blanchette VS. Immune thrombocytopenic purpura. N Engl J Med 2002; 346: 995-1008.

2. Cines DB, Bussel JB. How I treat idiopathic thrombocytopenic purpura (ITP). Blood 2005;106:2244-51.

3. Al-Moamary MS, Black W, Bessuille E, Elwood RK, Vedal S. The significance of the persistent presence of acid-fast bacilli in sputum smears in pulmonary tuberculosis. Chest 1999;116:726-31.

4. Madkaikar M, Ghosh K, Jijina F, Gupta M, Rajpurkar M, Mohanty D. Tuberculosis and immune thrombocytopenia. Haematologica 2002;87: ELT38.

5. Ghobrial MW, Albornoz MA. Immune thrombocytopenia: a rare presenting manifestation of tuberculosis. Am J Hematol 2001;67:139-43.

6. Tsuro K, Kojima H, Mitoro A, Yoshiji H,
Fujimoto M, Uemura M, et al. Immune thrombocytopenic purpura associated with pulmonary tuberculosis. Intern Med 2006;45: 739-42.

7. Brastanios PK, Swanson JW, Torbeson M, Sperati J, Karakousis PC. Tuberculosisassociated haemophagocytic syndrome. Lancet Infectious Disease 2006;6:447-54.

8. Kant S, Natu NK, Mahajan V. Rifampicin, ethambutol and pyrazinamide-induced thrombocytopenia. Int $\mathrm{J}$ Clin Pharmacol Ther 2008;46: 440-2. 